\title{
GASS: the Parkes Galactic all-sky survey
}

\section{Stray-radiation correction and second data release}

\author{
P. M. W. Kalberla ${ }^{1}$, N. M. McClure-Griffiths ${ }^{2}$, D. J. Pisano ${ }^{3, \star}$, M. R. Calabretta ${ }^{2}$, H. Alyson Ford ${ }^{2,5,9}$, F. J. Lockman ${ }^{4}$, \\ L. Staveley-Smith ${ }^{6}$, J. Kerp ${ }^{1}$, B. Winkel ${ }^{1}$, T. Murphy ${ }^{7,8}$, and K. Newton-McGee ${ }^{2,8}$
}

1 Argelander-Institut für Astronomie, Universität Bonn, Auf dem Hügel 71, 53121 Bonn, Germany e-mail: [pkalberla; jkerp; bwinkel]@astro.uni-bonn.de

2 Australia Telescope National Facility, CSIRO, Marsfield NSW 2122, Australia e-mail: [naomi .mcclure-griffiths;mark.calabretta]@csiro.au

3 Department of Physics, West Virginia University, PO Box 6315, Morgantown, WV 26506, USA e-mail: djpisano@mail.wvu.edu

4 National Radio Astronomy Observatory, Green Bank, WV 24944, USA e-mail: jlockman@nrao.edu

5 Centre for Astrophysics and Supercomputing, Swinburne University of Technology, Hawthorn VIC 3122, Australia

${ }^{6}$ International Centre for Radio Astronomy Research, M468, University of Western Australia, Crawley, WA 6009, Australia e-mail: Lister. Staveley-Smith@icrar.org

7 School of Physics, The University of Sydney, NSW 2006, Australia e-mail: tara@physics.usyd.edu.au

8 School of Information Technologies, The University of Sydney, NSW 2006, Australia e-mail: katherine.newton-mcgee@defence.gov.au

9 Department of Astronomy, University of Michigan, Ann Arbor, MI 48109, USA e-mail: haford@umich.edu

Received 28 December 2009 / Accepted 1 July 2010

ABSTRACT

\begin{abstract}
Context. The Parkes Galactic all-sky survey (GASS) is a survey of Galactic atomic hydrogen (HI) emission in the southern sky observed with the Parkes 64-m Radio Telescope. The first data release was published by McClure-Griffiths et al. (2009).

Aims. We remove instrumental effects that affect the GASS and present the second data release.

Methods. We calculate the stray-radiation by convolving the all-sky response of the Parkes antenna with the brightness temperature distribution from the Leiden/Argentine/Bonn (LAB) all sky 21-cm line survey, with major contributions from the 30-m dish of the Instituto Argentino de Radioastronomía (IAR) in the southern sky. Remaining instrumental baselines are corrected using the LAB data for a first guess of emission-free baseline regions. Radio frequency interference is removed by median filtering.

Results. After applying these corrections to the GASS we find an excellent agreement with the Leiden/Argentine/Bonn (LAB) survey. The GASS is the highest spatial resolution, most sensitive, and is currently the most accurate H I survey of the Galactic H I emission in the southern sky. We provide a web interface for generation and download of FITS cubes.
\end{abstract}

Key words. surveys - ISM: general - radio lines: ISM - Galaxy: structure

\section{Introduction}

Atomic hydrogen ( $\mathrm{HI})$ is ubiquitous, it traces the interstellar medium (ISM) in disk galaxies over a broad range of physical conditions. Its $21-\mathrm{cm}$ emission line is a key probe of the structure and dynamics of galaxies over a broad range of different densities and temperatures. $\mathrm{HI}$ is the key element to understand the evolution of the ISM in general (Kalberla \& Kerp 2009). 21-cm line observations of the Milky Way are of particular interest because small scale structures are observable which can not be resolved with observations of any other galaxy. A number of notable items have been detailed by McClure-Griffiths et al. (2009, hereafter Paper I), this includes the impact of massive stars on the ISM, the disk-halo interaction, the ISM life-cycle and the formation of cold clouds. H I is a perfect tracer of the

* Adjunct Assistant Astronomer at the National Radio Astronomy Observatory.
Galactic structure and there is a good correlation between gas and dust (Schlegel et al. 1998).

Galactic H I data are important for an exploration of the early universe because of the photoelectric absorption caused by the ISM in the soft X-ray energy range below $1 \mathrm{keV}$. The attenuation of the X-rays by the Galactic interstellar medium increases with decreasing X-ray energy (Kerp 2003). The emission of active Galactic nuclei at high redshifts $(z \sim 10)$ or the faint emission of clusters of galaxies at moderate redshifts $(3 \lesssim z \lessgtr 5)$ is shifted to this soft X-ray band. It is not possible to analyze the $\mathrm{X}$-ray data quantitatively without knowing in detail the distribution of the Galactic interstellar medium across the target.

High resolution single dish data are needed but neither the H I Parkes All Sky Survey (HIPASS) by Barnes et al. (2001) nor the reprocessed version (HIPASS-HVC) by Putman et al. (2002) provide accurate column densities for the Galactic emission in the southern sky. The GASS provides such data. For the northern sky the Galactic Arecibo L-Band Feed H I (GALFA-HI) survey 
A\&A 521, A17 (2010)

Table 1. Survey parameters for major single dish surveys of the Galactic 21-cm line emission. GALFA-HI and EBHIS are still in progress.

\begin{tabular}{|c|c|c|c|c|c|}
\hline Parameter & LAB & HIPASS-HVC & GASS & GALFA-HI & EBHIS \\
\hline Sky coverage & full sky & $\delta \lesssim 2^{\circ}$ & $\delta \lesssim 1^{\circ}$ & $-1^{\circ} \lesssim \delta \lesssim 38^{\circ}$ & $\delta \gtrsim-5^{\circ}$ \\
\hline Average beam $F W H M$ & $36^{\prime}$ & $14.4^{\prime}$ & $14.4^{\prime}$ & $3.4^{\prime}$ & $9.5^{\prime}$ \\
\hline LSR velocity range & $-450<v<+400$ & $-700<v<+1000$ & $-468<v<+468$ & $-750<v<+750$ & $-1000<v<+1000$ \\
\hline Channel width & $1.27 \mathrm{~km} \mathrm{~s}^{-1}$ & $26.4 \mathrm{~km} \mathrm{~s}^{-1}$ & $1.0 \mathrm{~km} \mathrm{~s}^{-1}$ & $0.18 \mathrm{~km} \mathrm{~s}^{-1}$ & $1.25 \mathrm{~km} \mathrm{~s}^{-1}$ \\
\hline $1 \sigma T_{\mathrm{B}}$ noise & $90 \mathrm{mK}$ & $24 \mathrm{mK}$ & $57 \mathrm{mK}$ & $\$ 100 \mathrm{mK}$ & $\$ 90 \mathrm{mK}$ \\
\hline
\end{tabular}

(Peek \& Heiles 2008) and the Effelsberg-Bonn H I Survey (EBHIS) are still in progress (Winkel et al. 2010). Major parameters for these surveys are compared in Table 1. Currently the LAB and the GASS are the only surveys that are easily accessible for data mining.

The Galactic All-Sky Survey (GASS) is a 21-cm line survey covering the southern sky for all declinations $\delta \lesssim 1^{\circ}$. The observations were made with the multibeam system on the 64-m Parkes Radio Telescope. The intrinsic angular resolution of the data is $14.4(F W H M)$. The velocity resolution is $1.0 \mathrm{~km} \mathrm{~s}^{-1}$ and the useful bandpass covers a velocity range $\left|v_{\mathrm{lsr}}\right| \lesssim 468 \mathrm{~km} \mathrm{~s}^{-1}$ for all of the observations; some data cover up to $\left|v_{\mathrm{lsr}}\right| \lesssim 500 \mathrm{~km} \mathrm{~s}^{-1}$. The GASS is the most sensitive, highest angular resolution largescale survey of Galactic H I emission ever made in the southern sky. The first data release is available at http://www. atnf. csiro.au/research/GASS.

A detailed description of the GASS project, focusing on the survey goals and techniques has been given by Paper I. In Paper I all of the initial data reduction and imaging is described in detail. Here we focus on the post-processing, in particular on corrections for stray-radiation, instrumental baselines, and radio frequency interference (RFI). We will note where our data reduction differs from that described in Paper I.

For 21-cm line observations there are a few unavoidable instrumental issues that can potentially degrade the data. The first is that a radio telescope does not have a perfect beam, so some radiation enters through the sidelobes of the antenna, causing spurious emission features. The second effect is that the receiver gain may drift, and it can suffer from instrumental spectral structure (the instrumental baseline) that may be timedependent. These influences need to be minimized, but the raw observations do not allow a clear identification and separation of individual instrumental problems. For example, spurious profile wings may be caused by stray-radiation but also by instrumental baseline defects. Temporal fluctuations in the line emission may be due to gain variations but may also be caused by spurious emission from the sidelobes.

A strategy is needed to disentangle these instrumental issues. For the stray-radiation correction a basic prerequisite is the knowledge of the H I brightness temperature distribution provided by the Leiden/Argentine/Bonn (LAB) 21-cm line survey (Kalberla et al. 2005); most important in the context of the GASS survey is the contribution from the $30-\mathrm{m}$ dish of the Instituto Argentino de Radioastronomía (IAR) in the southern sky (Bajaja et al. 2005). These surveys provide clean data with an angular resolution of $\sim 36^{\prime}$ and allow us to solve the Fredholm integral equation which describes the deconvolution of the raw observations (antenna temperatures) with respect to the influences of the antenna response. Bootstrapping from the LAB survey we use a model of the antenna response for the 13-beam receiver based on the known feed patterns and the telescope geometry. Section 2 describes how spurious features from the sidelobes are removed.

Next we remove baseline defects. For this the LAB survey is used for initial estimates of velocity ranges that do not contain line emission. In Sect. 3 we describe how baselines are refined in an iterative way. A gain calibration requires some preliminary baseline corrections but such a calibration also affects the accuracy of the corrections for stray-radiation. The calibration is described in Sect. 4. All corrections discussed in Sects. 2 to 4 need to be iterated several times.

RFI is increasingly a problem for sensitive observations, and post-processing mitigation strategies are essential. We use flags which were set in the first stage of the data processing by Livedata described in Paper I and iteratively remove the remaining interference as discussed in Sect. 5. Throughout the reduction we take care that the corrections described in Sects. 2 to 4 are not affected by RFI.

In comparison with the LAB survey, the GASS represents an improvement in velocity resolution and sensitivity, but most important is the enhanced spatial resolution (Table 1). The generation of FITS images with user-definable parameters is described in Sect. 6. We discuss some examples of the newly corrected data in Sect. 7 and complete the paper with a description of a web interface for download (Sect. 8).

\section{The stray-radiation correction}

A major problem when observing 21-cm emission lines is caused by the fact that Galactic emission is seen in all directions. Most prominent is the bright Galactic plane which extends across the whole sky. It is unavoidable that stray 21-cm emission from the plane will get picked up by sidelobes of the antenna. The main sidelobes are the result of reflections from the feed legs that carry the prime focus cabin. Also radiation originating from regions outside the rim of the reflector, the spillover region, is received. Stray-radiation varies with time (van Woerden 1962) and for positions with very low H I the spurious emission features may amount to $50 \%$ of the total received signal (Kalberla et al. 1980a; Lockman et al. 1986; Hartmann et al. 1996). In some GASS spectra stray-radiation was found to comprise $35 \%$ of the observed emission.

Stray-radiation may not be neglected if we are interested in low-intensity profile wings that may originate from lines with large velocity dispersion, or in deriving accurate H I column densities from $21-\mathrm{cm}$ observations. Observed lines may originate either from the main beam, the "real" signal, or may have been picked up from other directions, the "stray" signal. Without prior knowledge of instrumental issues it is also very difficult to distinguish between faint line emission and instrumental baseline defects.

One way to minimize stray-radiation is by minimizing scattering surfaces in the telescope aperture. The optimal case appears to be a telescope constructed with a clean unblocked aperture (Dickey \& Lockman 1990). Prime examples are the Bell Labs horn reflector antenna (Stark et al. 1992) and the Green Bank Telescope (GBT; Prestage et al. 2009). A typical parabolic reflector antenna has a main beam efficiency of $70 \%$, for unblocked apertures this improves to $\gtrsim 90 \%$. Sidelobe contributions 
are thus reduced from $30 \%$ to $10 \%$ but are still recognizable (Lockman \& Condon 2005). Depending on the observed position, emission from the spillover region may cause severe contaminations in the case of an unblocked aperture with a receiver in the secondary focus (Robishaw \& Heiles 2009).

A numerical solution of the stray-radiation problem was proposed by van Woerden (1962). He demonstrated convincingly that the stray component can be determined by convolving the antenna pattern with the brightness temperature distribution on the sky. Yet, neither the knowledge about sidelobe structures, nor the computing power was sufficient enough for accurate calculations in 1962. The first reliable calculations became available for the Effelsberg telescope (Kalberla et al. 1980a,b), and later for the resurfaced Dwingeloo dish (Hartmann et al. 1996), the 26-m Telescope at the Dominion Radio Astrophysical Observatory (Higgs \& Tapping 2000), and the 30-m telescope at Villa Elisa (Bajaja et al. 2005). A refined correction of the Leiden/Dwingeloo survey (Hartmann \& Burton 1997) in combination with the Villa Elisa observations led to the LAB survey (Kalberla et al. 2005). Currently the LAB is considered to be the most accurate Galactic all-sky survey.

\subsection{Basics}

We use a correction algorithm developed by Kalberla et al. (1980a). Antenna temperatures $T_{\mathrm{a}}$ observed by radio telescopes can be approximated in Cartesian coordinates as a convolution of the true temperature distribution $T$ on the sky with the beam pattern $P$ of the antenna

$T_{\mathrm{a}}(x, y)=\int P\left(x-x^{\prime}, y-y^{\prime}\right) T\left(x^{\prime}, y^{\prime}\right) \mathrm{d} x^{\prime} \mathrm{d} y^{\prime}$.

This is an approximation, but is sufficient to demonstrate the basics. In general, $T_{\mathrm{a}}$ is time and frequency dependent, spherical coordinates should be used and the integration needs to be extended over the observable part of the sky as well as the ground if there are reflections. We do not include in these equations the effects of atmospheric attenuation, though this must be considered for emission entering both the main beam and the sidelobes (Lockman 2002).

For the pattern $P$ of the antenna we use the normalization

$\int P(x, y) \mathrm{d} x \mathrm{~d} y=1$

in addition, we split the pattern into the main beam area (MB) and the stray-pattern (SP)

$$
\begin{aligned}
T_{\mathrm{a}}(x, y)= & \int_{\mathrm{MB}} P\left(x-x^{\prime}, y-y^{\prime}\right) T\left(x^{\prime}, y^{\prime}\right) \mathrm{d} x^{\prime} \mathrm{d} y^{\prime} \\
& +\int_{\mathrm{SP}} P\left(x-x^{\prime}, y-y^{\prime}\right) T\left(x^{\prime}, y^{\prime}\right) \mathrm{d} x^{\prime} \mathrm{d} y^{\prime} .
\end{aligned}
$$

Defining the main beam efficiency $\eta_{\mathrm{MB}}$ of the telescope as

$\eta_{\mathrm{MB}}=\int_{\mathrm{MB}} P(x, y) \mathrm{d} x \mathrm{~d} y$

we may rewrite Eq. (3) as

$T_{\mathrm{B}}(x, y)=\frac{T_{\mathrm{a}}(x, y)}{\eta_{\mathrm{MB}}}-\frac{1}{\eta_{\mathrm{MB}}} \int_{\mathrm{SP}} P\left(x-x^{\prime}, y-y^{\prime}\right) T\left(x^{\prime}, y^{\prime}\right) \mathrm{d} x^{\prime} \mathrm{d} y^{\prime}$.

Replacing $T_{\mathrm{B}}$ by $T$ leads to a Fredholm integral equation of the second kind which can be solved for $\eta_{\mathrm{MB}}>0.5$
(Kalberla et al. 1980a). $T_{\mathrm{B}}$ is the so-called brightness temperature, resulting from a convolution between the main beam of the telescope and the true sky temperature $T$. A solution of Eq. (5) is simplified considerably for a known all-sky brightness temperature distribution $T$. We use profiles from the LAB survey as the currently best possible approximation to $T$. For a solution of Eq. (5), $P$ needs to be determined. This is described in Sects. 2.3 and 2.4 .

\subsection{The correction algorithm}

The numerical correction algorithm is based on Kalberla et al. (1980a). It was originally developed for the Effelsberg 100-m telescope, with some improvements it was then applied to the LAB survey (Kalberla et al. 2005). We modified this procedure for use with multi-beam systems, allowing individual corrections for each of the beams. Due to the rotation of the offset receivers with respect to the telescope structure, we take into account the variation of each receiver's beam pattern on the sky with time. To improve the accuracy of the calculations we also changed the convolution algorithm for the inner part of the antenna response pattern (within $6^{\circ}$ of the main beam). From the LAB survey, a new and more accurate input sky for the convolution (Eq. (5)) was generated.

\subsection{The multi-beam antenna pattern - near sidelobes}

To solve Eq. (5) it is necessary to know the complete antenna response $P$ for each of the 13 beams of the Parkes multi-feed system. In practice there are severe limitations in measuring the sidelobes to the required accuracy. We have therefore chosen to model the sidelobes. In general, the far-field pattern of an antenna is the autocorrelation of the aperture plane distribution. Using the measured feed response pattern and the telescope geometry, including shadowing caused by the focus cabin and the feed support legs, we derive the complex aperture distribution function for each feed. The pattern can then be calculated by Fourier transformation.

For the 13 beams we distinguished three characteristic cases, the central beam and one feed from the inner and outer ring of feeds (Fig. 1 top). The feed horns are equipped with two pairs of orthogonal-linear probes, which, while in parallactic tracking, rotate relative to the feed support legs. In turn the complex aperture distribution function changes with time. We approximate this rotation with an accuracy of $\pm 5^{\circ}$. The fact that the three feed support legs cause a global six-fold symmetry for the antenna pattern simplifies the situation considerably. We need only to calculate 5 individual patterns for each of the rings with radial feed offsets of 29'.1 and 50'.8, respectively.

Figure 1 shows the feed configuration for the multibeam system ${ }^{1}$ and displays the antenna response pattern within a radius of $6^{\circ}$ for feeds 1,2 , and 8 . We see characteristic changes due to the beam displacements. The main beam develops a coma lobe that increases in strength with feed offset. Sidelobe structures become asymmetric and have a more pronounced ringing with increasing feed offset. An important feature of the multifeed system is that the azimuthal asymmetries in the antenna pattern due to the feed offsets decrease on average with increasing distance from the main beam. Extrapolating this property to far sidelobes, offset from the main beam by tens of degrees, implies that differences between individual feeds should vanish. Indeed we verified this behavior as described in Sect. 2.4.

\footnotetext{
1 http://www.atnf.csiro.au/research/multibeam/.overview.html
} 


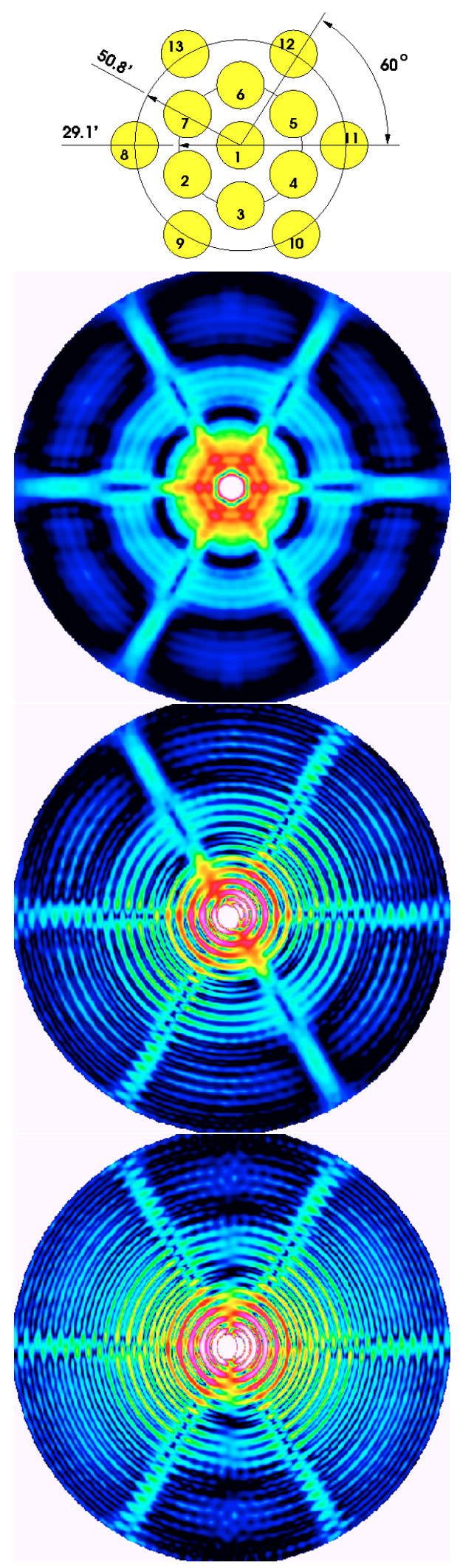

Fig. 1. Feed configuration for the receiver (top) and antenna patterns within a radius of $6^{\circ}$ for beam 1,2 , and 8 (from top to bottom). The display is restricted to levels between $-20 \mathrm{~dB}$ (white) to $-50 \mathrm{db}$ (black).

In the correction algorithm it is not necessary to use a pattern with all the details shown in Fig. 1 as the LAB data were measured on a grid of only $0.5 \times 0.5$. We have therefore modeled the inner 6.0 of the antenna patterns on a grid containing 468 cells in cylindrical coordinates. The cells have an azimuthal extent of $10^{\circ}$ and a radial extent of 0.2 out to 2.2 from the main beam, a radial extent of 0.3 out to 2.5 , and of 0.5 thereafter.

\subsection{The far sidelobes}

The far sidelobes arise predominantly by reflections from the feed support legs, causing the so-called stray-cones, and at the rim of the telescope, the spillover. The location of these structures on the sky was determined from the telescope geometry and verified by numerous tests made with a transmitter. The sidelobe levels from the spillover lobes were estimated from the edge taper of the receiver feed. For the stray-cones we used estimates from the the aperture blocking by the feed legs. These estimates were also based on sidelobe levels found previously for the telescopes at Effelsberg, Dwingeloo and Villa Elisa (Kalberla et al. 1980a; Hartmann et al. 1996; Bajaja et al. 2005; Kalberla et al. 2005). The far sidelobe components were adjusted individually in a way similar to that described by Kalberla et al. (2005), searching for a consistent solution of Eq. (5).

\subsection{Ground reflections}

Sidelobes that touch the ground receive broad-band thermal noise, but to first order no line radiation is expected. However, depending on the ground cover, reflections may happen. While scrub acts as an absorber, low grass and soil can reflect $21-\mathrm{cm}$ line radiation. We determine this contribution by surveying the ground around the telescope, and then using ray-tracing methods to estimate the effective sidelobe response. We used an albedo of 0.2 .

\section{Removal of instrumental baselines}

\subsection{Preliminary considerations}

In many cases, stray-radiation produces extended profile wings that mistakenly may be interpreted as instrumental baseline structure. A correction for stray-radiation could cause negative intensities if applied after the baseline correction. It is therefore essential to apply baseline corrections only after the strayradiation is eliminated. For this reason we had to deviate from the processing described in Paper I; for the first data release no correction for stray-radiation was applied. We used Livedata as described in their Sect. 2.3 for the bandpass correction, but without the post processing for baselines according to Sect. 2.3.3 in Paper I. The algorithm described below is in lieu of the postprocessing described in Paper I.

After scaling the reduced data to antenna temperatures corrected for atmospheric absorption (Sect. 4) the stray-radiation was subtracted and afterwards the instrumental baseline was corrected. Flags set by Livedata or our own software to indicate the presence of RFI were taken into account, and affected channels were disregarded for all of the baseline fits described below.

\subsection{The algorithm}

For each spectrum of the stray-radiation-corrected GASS database we used the LAB survey for an initial estimate of the velocity extent of the expected $\mathrm{HI}$ emission. After smoothing the interpolated $\mathrm{LAB}$ profile to an effective resolution of $\Delta v_{1 / 2}=5 \mathrm{~km} \mathrm{~s}^{-1}$, we used velocities where $T_{\mathrm{LAB}}<0.9 \mathrm{~K}$ as the range for an initial fit of the instrumental GASS baseline. In 
addition we folded the LAB profile in frequency to mimic the second spectrum used by Livedata to determine the instrumental bandpass (Paper I, Fig. 3). Flags were transferred accordingly.

GASS spectra from both polarizations were averaged and smoothed to $\Delta v_{1 / 2}=5 \mathrm{~km} \mathrm{~s}^{-1}$ for each of the individual $5 \mathrm{~s}$ dumps. Using the initial estimate for the emission-free part of the profile we determined the instrumental baseline by fitting a 9th order polynomial. Any channel with signal exceeding four times the rms noise over the fitted region was considered to contain possible $\mathrm{HI}$ emission and was then excluded from the baseline region. The baseline fit was repeated with an 11th order polynomial, again searching for potential emission that needed to be excluded from the fit. As a measure of the fit quality we determined the residual rms scatter within the baseline region. For a few percent of the data we found that a 9th order polynomial provided a better fit. Accordingly we used this fit. Finally we took into account that weak profile wings with intensities below the automatic $4 \sigma$ cut-off level could affect the fit parameters. To avoid such a bias we excluded 6 more channels on each side of the H I emission from the baseline region that was fitted.

After these initial steps we considered both spectra for each polarization individually. The spectra were smoothed and fitted with the parameters determined previously to find out whether some more features needed to be excluded from the baseline region. After that the final baseline fit was applied to the original observations.

The algorithm and the parameters described above were tested and optimized by varying the polynomial order and trying other functional forms. We particularly tried to avoid high order polynomials. As described in Paper I, the average quotient spectrum for each of the scans (visible as patches in Fig. 9, bottom) was fitted with a 15 th order polynomial. Figure 3 of Paper I demonstrates that emission features are not affected by this fit. After this global bandpass fit there remain some time dependent fluctuations that need to be corrected independently for each spectrum with $5 \mathrm{~s}$ integration time. A first guess would be that a similar polynomial is needed to get rid of such fluctuations. This is fortunately not the case. In the initial Livedata reduction for the first data release a 10th order fit for post-processing of individual spectra was sufficient. Our results confirm this finding with minor modifications for our procedure. We tested lower order polynomials. These led for many regions to obvious baseline defects. We found spurious features in emission free regions that could only be avoided by allowing higher orders.

Assuming that some parts of the residual baseline ripples could be caused by standing waves, we also tested sine wave fits in addition to polynomials. Sinusoid solutions were found to be insignificant and were discarded. We carefully checked whether errors in the LAB data might affect the GASS baseline correction, but the iterative refinements that were applied successfully avoided any biases. We tested also whether the high order polynomial fits might have affected weak emission features. We found no indications for such problems. In particular the RFI feature observed in March 2006, appearing similar to a HVC (Sect. 5.5), remained unaffected by the fit. Also testing whether profile wings in data that remained uncorrected for stray-radiation could be removed by baseline fitting did not indicate an unwanted overcorrection by the baseline fit (see Sect. 7).

Some residual systematical baseline problems remain in a few regions, on average typically at a level of $\$ 40 \mathrm{mK}$ but occasionally up to $100 \mathrm{mK}$. This is close to the rms uncertainties of $\sim 60 \mathrm{mK}$ for individual maps as discussed in Sect. 7 . We were unable to identify the reason for such systematic baseline errors.
Table 2. $T_{\mathrm{B}}$ calibration positions and parameters.

\begin{tabular}{cccccc}
\hline \hline Pos. & $l$ & $b$ & $\begin{array}{c}\text { Peak } T_{\mathrm{B}} \\
(\mathrm{K})\end{array}$ & $\begin{array}{c}\text { Area } \\
\left(\mathrm{K} \mathrm{km} \mathrm{s}^{-1}\right)\end{array}$ & $\begin{array}{c}\text { Vel. range } \\
\left(\mathrm{km} \mathrm{s}^{-1}\right)\end{array}$ \\
\hline S8 & 207.00 & -15.00 & 75.6 & 879.5 & -5.1 to +22.3 \\
S9 & 356.00 & -4.00 & 83.5 & 930.8 & -1.5 to +15.2 \\
S6 & 1.91 & $41^{\circ} .42$ & 51.0 & 285.5 & -5.8 to +4.8 \\
\hline
\end{tabular}

They may be caused by RFI or bad fits but also by uncertainties in the stray-radiation correction.

\section{Calibration}

An accurate correction for stray-radiation demands a careful calibration. Errors in the antenna temperature scale cause a mismatch with the stray-radiation correction term in Eq. (5). The sidelobe response is frequency dependent; calibration errors, therefore, cause not only an increased scale error but also affect the profile shape. We used regular observations at the standard positions S8, S9, and S6 (Williams 1973) and determined average calibration factors for each of the observing sessions. An initial temporary calibration was determined from uncorrected data (Paper I). After correcting the data for stray-radiation we redetermined the $T_{\mathrm{B}}$ calibration factors and applied these new factors for the following calculations.

Table 2 lists the final calibration data; the brightness temperature scale is matched to a common temperature scale first established by Williams (1973). The brightness temperature for most of the major surveys are matched through this reference; this includes the Hat Creek surveys (Weaver \& Williams 1973; Heiles \& Habing 1974), the Leiden-Green Bank survey (Burton 1985), southern sky surveys by Kerr et al. (1981), the Bell Labs survey (Stark et al. 1992), the LAB survey, and Effelsberg and Arecibo data (Peek \& Heiles 2008; Winkel et al. 2010).

A few calibration regions have been mapped by Kalberla et al. (1982). They calculated the beam response in detail and provided also calibration factors as a function of the beam shape. We use factors appropriate for a $14.4^{\prime}$ beam.

The easiest way to determine the calibration factors is to use peak temperatures, but line integrals over a limited velocity range are more accurate (Williams 1973) and we preferred this method. Please note that velocities listed in Table 2 are integration limits while velocities given by Williams (1973) are center velocities for the edge channels (see Kalberla et al. 1982). Figure 2 (left) compares overall gain factors applied to the initial calibrations (blue) with those after completing the final corrections (red and green). The overall calibration uncertainties (right) for most of the feeds could be reduced to typically $1.7 \%$. Only two receivers were less stable with an $\mathrm{rms}$ scatter of $3 \%$ and $3.5 \%$. The uncertainties are of statistical nature. Combining observations with several beams at different seasons we expect average temperature scale uncertainties well below $1 \%$ in the final data. We note that interference can cause a loss of calibration in some spectra that would be difficult to detect as it would appear as a scale factor over an entire spectrum. We believe that this effect is small in the overall survey but may be present in some final spectra.

\section{RFI mitigation}

\subsection{Replacement of data flagged by Livedata}

Narrow-line interference was flagged during the basic data reduction with Livedata (Paper I, Sect. 2.3.2). Most of this type of 

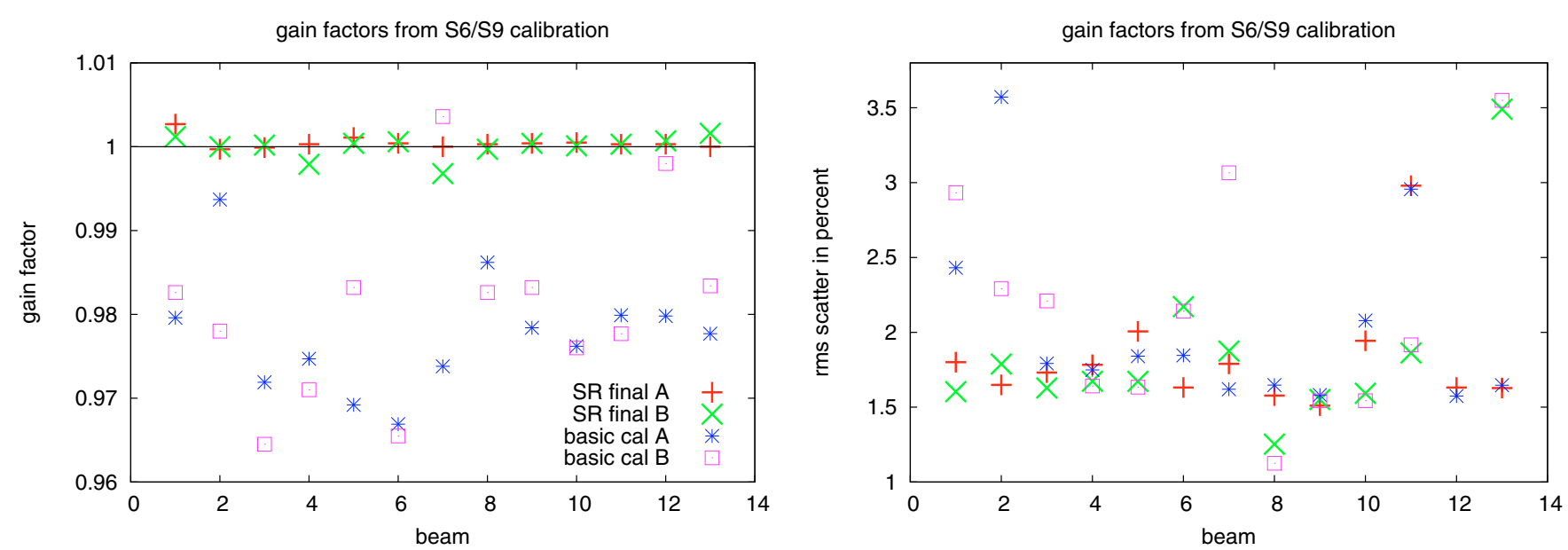

Fig. 2. Left: gain factors applied to the initial calibration (blue stars and purple squares) and cross check after the final data reduction (red plus signs and green X's). Right: rms scatter in gain calibration for individual feeds, before and after correction for stray-radiation (same symbols as in left panel).
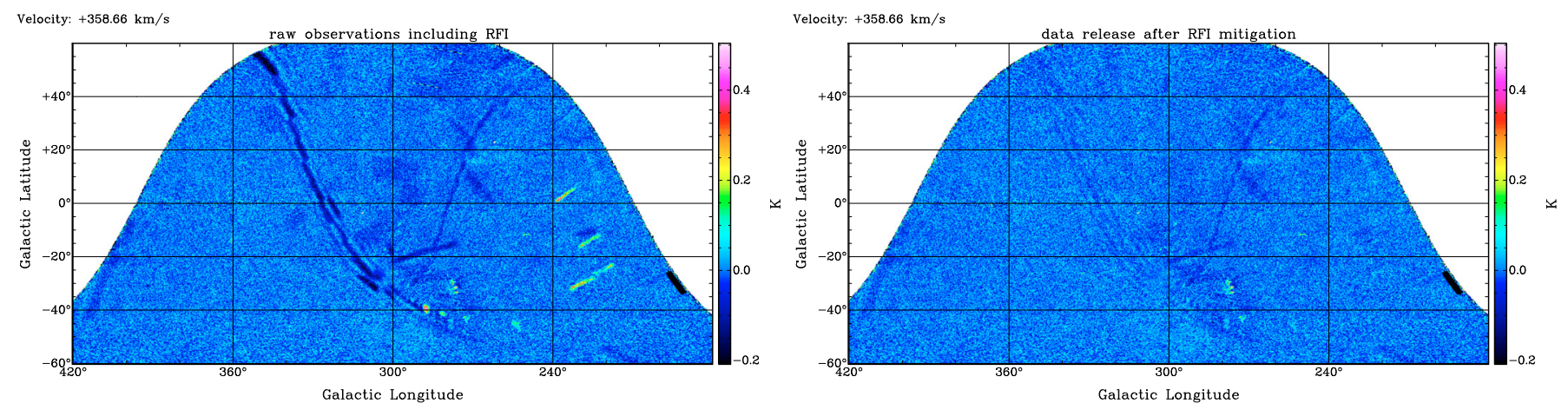

Fig. 3. Typical example for extended interference patterns that were flagged by Livedata in the first stage of the data reduction. Left: as observed, right: after RFI removal using LAB data as described in Sect. 5.1. The intensity scale is linear for $-0.2<T<0.5 \mathrm{~K}$. Some weak structures remain but are mostly at $|T|<40 \mathrm{mK}$.

RFI occurs at a fixed topocentric frequency, so as the telescope scans across the sky the RFI moves in successive channels in the LSRK frame. We use the kinematical (radio) standard of rest definition with a velocity of $20 \mathrm{~km} \mathrm{~s}^{-1}$ in direction Ra, Dec = $\left[270^{\circ},+30^{\circ}\right]$ (epoch 1900.0). Most of the features are observed to stretch over large angular distances and affect fields that have been observed independently at different seasons (Fig. 3). This behavior implies that the RFI remained approximately constant for long periods in time, affecting most of the data.

Profiles with more then 30 flagged channels were completely discarded during imaging; for less serious contaminations the flagged data were replaced. After correcting profiles for strayradiation and instrumental baseline, we interpolated LAB survey data to replace the flagged channels. Alternatively we have tried to replace data by interpolating the GASS spectra using the nearest ten good data points. Using LAB data resulted in all cases in much cleaner maps. Still, some weak stripy features remain that appear to move across the sky in successive velocity channels but mostly these artifacts remain within the noise (Fig. 3). About $0.1 \%$ of all data have been flagged by Livedata and were replaced by interpolated data in this first step. We kept the Livedata flags to avoid such interpolated data getting propagated by the RFI mitigation described in the following sections.

\subsection{Median filter RFI rejection}

Even after removal of the channels flagged in the initial data reduction, numerous instances of RFI remain in the data. Some appear as point sources, but the most obvious show "footprints" of the 13-beam system that move on the sky in scanning direction (Fig. 4). As before, the motion in successive velocity channels is caused by changing LSR projections of the fixed Topocentric RFI frequencies. Features of this kind were found previously in the HIPASS and are described in detail by Barnes et al. (2001, Fig. 7). For the first data release the remaining RFI was removed by median gridding when calculating FITS images (Paper I, Sect. 2.3.5).

Here we follow a different strategy, taking advantage of the fact that in GASS typically 40 individual spectra contribute to the final data in every resolution element. First, for any observed position in the sky we selected all observed profiles within an angle $\epsilon$ of typically a few arc-minutes. From these data we calculate the mean for each channel, the corresponding standard deviation, and the median, disregarding data flagged either by Livedata or our own post-processing. The average rms noise level $T_{\mathrm{rms}}=\sigma(T)$ and its one sigma deviation $\sigma\left(T_{\mathrm{rms}}\right)$ from the average was determined for the low level emission part of the profile $\left(T_{\mathrm{B}}<0.5 \mathrm{~K}\right)$.

We have two tests for RFI. In the first, channels with rms deviations $\Delta T_{\mathrm{rms}}>3 \sigma\left(T_{\mathrm{rms}}\right)$ were considered as highly suspicious for RFI contamination and investigated in more detail. We use as a second indication of RFI circumstances where the mean and median differ from each other by more than one standard deviation. 

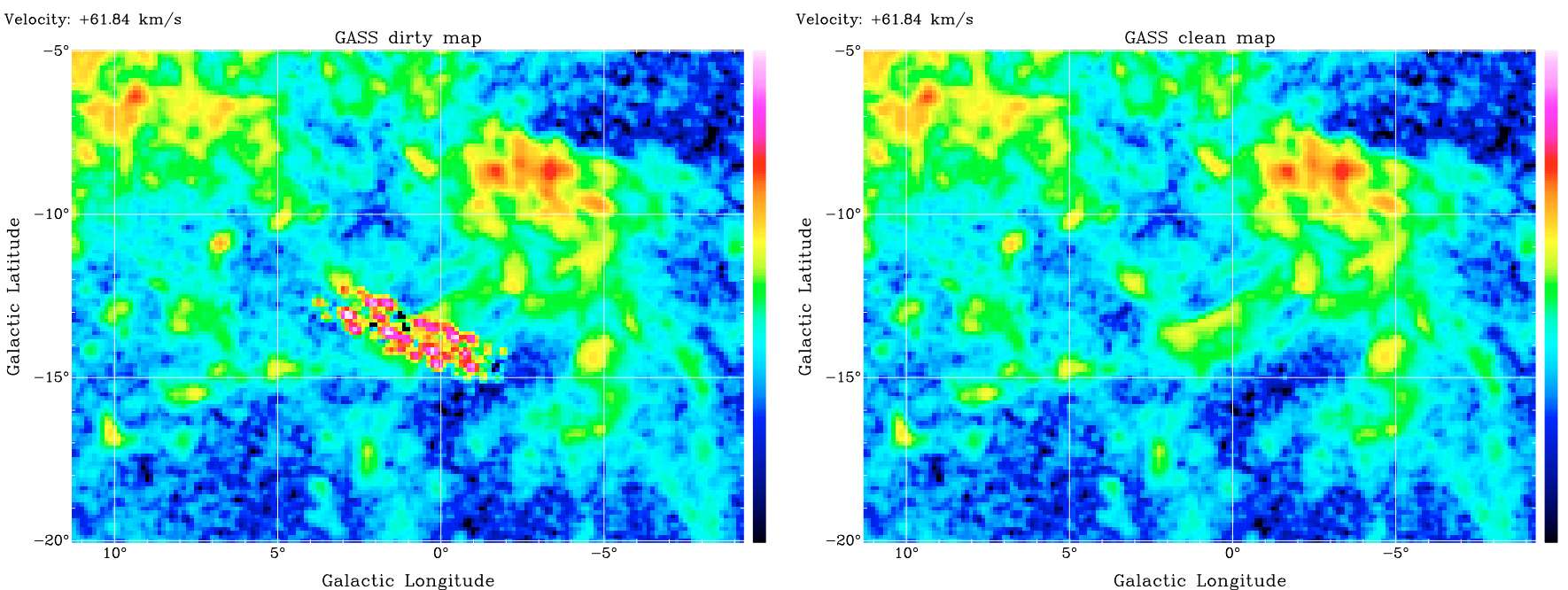

Fig. 4. HI emission at $v_{\mathrm{lsr}}=61.8 \mathrm{~km} \mathrm{~s}^{-1}$. Data heavily affected by RFI in scanning direction of the telescope (left) are compared with the clean map (right). The intensity scale for the range $-.04<T<20 \mathrm{~K}$ is logarithmic, emphasizing low level emission. Yellow colors correspond to a brightness temperature of $\sim 1 \mathrm{~K}$.

To eliminate outliers for the channels that were selected in both of the two tests we determined if individually observed brightness temperatures deviate from the median by more than two times the standard deviation. In the case of a purely random distribution this would exclude $5 \%$ of the good data, and we consider this an acceptable cost to eliminate true RFI. We flag outliers and repeat, calculating new mean and median estimators and once more excluding any outliers.

Our two sigma limit may be compared with the more rigorous method for the elimination of suspect data proposed by Peirce (1852) which is based on probability theory. Accordingly a rejection of a data point that deviates from the mean at a two sigma level is justified if there are 13 independent observed data values. For $\epsilon=6^{\prime}$ (see below) we have on average 40 individual profiles and a two sigma limit is appropriate if about $10 \%$ of the data are suspect. This condition is valid for many cases but we find also situations where $20 \%$ of the measurements are outliers. In this case a cutoff at $1.6 \sigma\left(T_{\text {rms }}\right)$ would be more appropriate according to Peirce (1852). We deviate from Peirce's criterion in two ways; to simplify the calculations we use a fixed $2 \sigma\left(T_{\text {rms }}\right)$ cutoff and we define outliers by their deviations from the median and not, as originally proposed by Peirce, from the mean. The latter is essential since outliers caused by RFI can be found at ten to hundred times the standard deviation, a circumstance that was not considered by Peirce; in such cases the mean is usually seriously affected.

To replace values outside the two sigma range, the best available estimate is the median value scaled by the proper beam weighting function, for details we refer to the extended discussion by Barnes et al. (2001, Sect. 4.2.2). We replace the previously selected data points by the weighted median and set a flag to distinguish medians from observed values. A fraction $\sim 5 \times 10^{-4}$ of the data is affected by RFI and replaced by the weighted median.

One of the criteria that we used to detect RFI was the fact that mean and median should not differ by more than one standard deviation. We applied this criterion also to test whether the median estimator is consistent with the mean of all data after exclusion of the outliers. Both methods were found to produce nearly identical results. An example of RFI in scanning direction and the result after application of our median filer is given in Fig. 4.

Figure 5 shows spectral details for one position in this field. Displayed are the mean (red), the rms scatter (green), and the median (blue) derived for all profiles that are closer than $\epsilon<6^{\prime}$ to the position $l=0^{\circ}, b=-13.5$. Data in the left plot suffer from strong interference at $v_{\text {lsr }} \sim 157 \mathrm{~km} \mathrm{~s}^{-1}, 55 \lesssim v_{\text {lsr }} \lesssim 58 \mathrm{~km} \mathrm{~s}^{-1}$, and at $v_{\text {lsr }} \gtrsim 400 \mathrm{~km} \mathrm{~s}^{-1}$. Only a part of the data was flagged by Livedata and replaced by values from the LAB. Despite all defects the median (blue) is essentially unaffected by RFI. The data after RFI elimination are shown in the plot at the right side. Profiles for mean and median are within the noise identical, the rms is close to the expected value of $0.4 \mathrm{~K}$ and shows no strong enhancements. We find some fluctuations close to the line emission at $v_{\mathrm{lsr}} \sim 0 \mathrm{~km} \mathrm{~s}^{-1}$. This may have been caused by genuine fluctuations in the H I line emission. To avoid a possible degradation of such fluctuations no automatic RFI cleaning was applied for $T_{\mathrm{B}} \gtrsim 0.5 \mathrm{~K}$. These two plots demonstrate how greatly the rms is affected by RFI, and how deviations between median and mean are obvious. Our RFI filter is triggered by such deviations.

\subsection{The beam-specific parameter $\epsilon$}

A critical parameter for RFI filtering is the radius $\epsilon$ for a comparison of the data at adjacent positions. A large value provides a large statistical sample but at the same time point sources or steep gradients in the brightness distribution may be affected by the filter. We found in tests that $\epsilon=6^{\prime}$ is sufficiently robust. Tests with $\mathrm{H}$ I point-sources have shown that larger values could degrade the source distribution. Our result is in excellent agreement with Barnes et al. (2001), and our median estimator is identical with the parameter $r_{\max }=6^{\prime}$ that was used for HIPASS.

\subsection{RFI in emission line regions}

RFI that falls in channels containing H I emission was usually removed successfully by the above procedure, except in regions with strong spectral gradients. Examples of such critical cases 

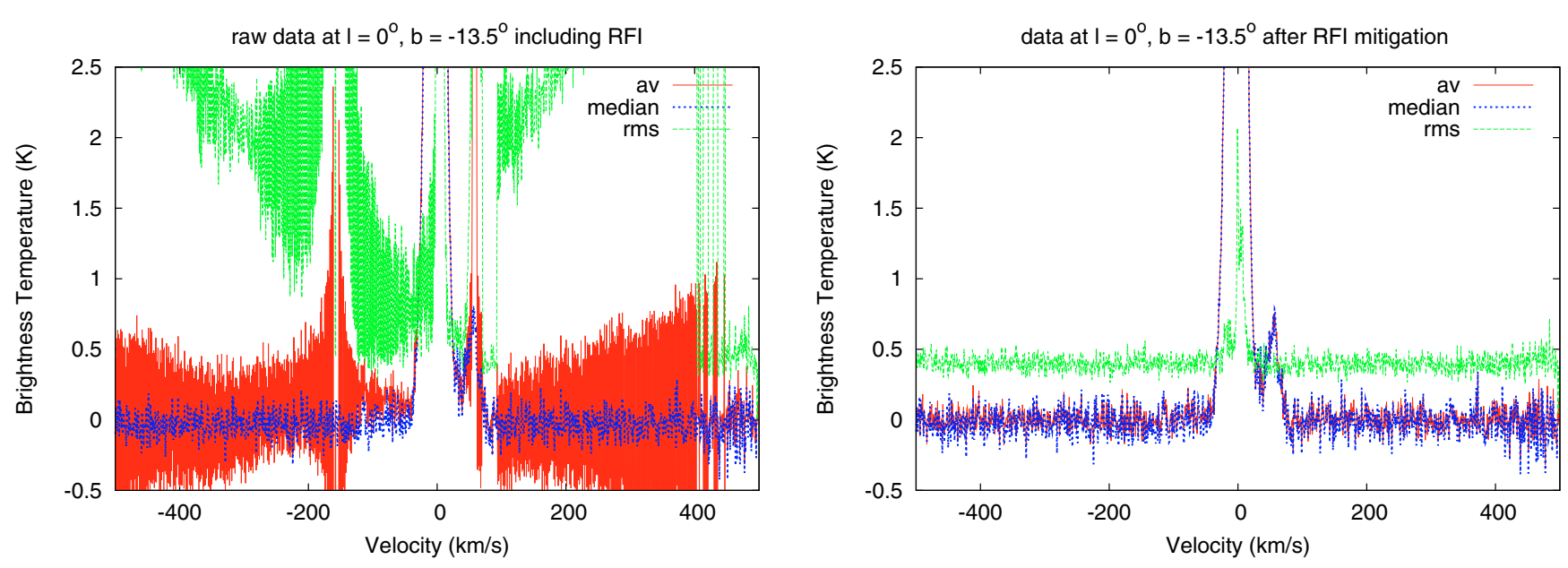

Fig. 5. Data at the position $l=0^{\circ}, b=-13.5$ before and after RFI mitigation. The average is plotted in red, the rms scatter in green, and the median in blue.

are positions with strong absorption lines. Sources with a continuum flux exceeding $200 \mathrm{mJy}$ were excluded from RFI rejection.

To avoid any possible degradation of the signal due to an automatic RFI filtering, the median filters described in Sect. 5.2 were restricted to data with low brightness temperatures. We used a limit of $T_{\max } \lesssim 0.5 \mathrm{~K}$. The remaining data were checked visually. Only a few cases showed some residual RFI. For these we increased the filter limits such that only channels with sufficient large rms deviation $\Delta T_{\text {rms }}$ were affected. We verified that the remaining data were unaffected by the median filter. As a general rule we tried to avoid as far as possible any modification of the H I emission lines through the RFI filtering process. This implies, however, that some RFI may remain undetected and be present in the final data.

Some of the spectra were affected so severely by RFI that they needed to be removed completely. We eliminated all profiles whose mean rms within the baseline region exceeded the average noise level at its position by a factor of three. About $0.3 \%$ of the observed profiles were rejected for this reason.

\subsection{RFI in March 2006}

For a short period between 18 and 21 March 2006 observations were degraded by broadband RFI with quite different characteristics than any of the narrow band RFI described above. The weak spurious lines had approximately Gaussian shapes with center velocities in the range 270 to $310 \mathrm{~km} \mathrm{~s}^{-1}$ and velocity dispersions of about $15 \mathrm{~km} \mathrm{~s}^{-1}$. None of the RFI rejection methods described above applied to this case. These spurious lines were identified by fitting Gaussians; all affected channels were then flagged. Baseline corrections were repeated, finally all flagged data were replaced by medians as described before. This way we recovered data that were discarded in the first data release.

\subsection{Removal of bandpass ghosts}

To maximize sensitivity and recover all the extended H I emission, the GASS was made using in-band frequency-switching with an offset of $3.125 \mathrm{MHz}$ corresponding to $660 \mathrm{~km} \mathrm{~s}^{-1}$. The Livedata bandpass correction causes for every real emission line feature an associated, spurious, negative image ("ghost") in the other band, displaced by $\pm 660 \mathrm{~km} \mathrm{~s}^{-1}$. Most of these negative images fall outside the velocity coverage of the survey but high velocity clouds (HVCs) with emission lines at $\left|v_{\mathrm{lsr}}\right| \gtrsim 160 \mathrm{~km} \mathrm{~s}^{-1}$ cause ghosts. In particular, the Magellanic System causes a strong and extended bandpass ghost at negative velocities.

As Fig. 3 of Paper I shows, ghosts appear only in one of the two IFs; the other band is unaffected. It is possible to produce maps without ghost features if one uses only one IF for $\left|v_{\text {lsr }}\right| \gtrsim 160 \mathrm{~km} \mathrm{~s}^{-1}$. A second possibility is to flag ghosts and eliminate them with a treatment analogous to one of the the RFI mitigation strategies discussed above. Accordingly the ghosts were replaced by medians determined from the alternate ghostfree IF band. Such maps have the advantage that they provide a better sensitivity for most of the field but remaining biases can not be excluded. We determined for both bands all HVC emission features with $\left|v_{\text {lsr }}\right| \gtrsim 160 \mathrm{~km} \mathrm{~s}^{-1}$ and $T_{\mathrm{B}}>0.2 \mathrm{~K}$ (corresponding to $3 \sigma\left(T_{\mathrm{rms}}\right)$ for $\left.\epsilon=6^{\prime}\right)$ and flagged ghosts in the other band. These features were treated analogously to the broadband RFI discussed in the previous section.

\section{Imaging}

For HIPASS, as well as for the first data release of the GASS, imaging was performed by Gridzilla using weighted median estimation which is robust against RFI and other artifacts. For the second data release, we use separate and independent procedures for RFI rejection and imaging. As described in the previous section, our median filter is largely consistent with the HIPASS median filter algorithm for a radius $\epsilon=6^{\prime}$. However, the major difference is that we apply this filter only to individual channels if two conditions apply: 1) an exceedingly large scatter is observed with a 3 times larger dispersion than the mean dispersion caused by thermal noise and 2) mean and median differ by more than one standard deviation. Only data that deviate from the median by $\Delta T_{\mathrm{rms}}>2 \cdot \sigma\left(T_{\mathrm{rms}}\right)$ are replaced by the median estimate.

For the data release described here the gridding was performed using a two-dimensional Gaussian with a user-definable dispersion. For computational reasons we disregard data outside a three sigma cutoff radius. The interpolated value for that pixel is the weighted mean over all available input data. Except for the irregular distribution on the sky of the input data, this interpolation is equivalent to a linear convolution of the observed brightness temperature distribution with the kernel function of the gridder. The resulting effective beam is approximately Gaussian. For a source width $W_{\mathrm{s}}$, an effective average telescope 


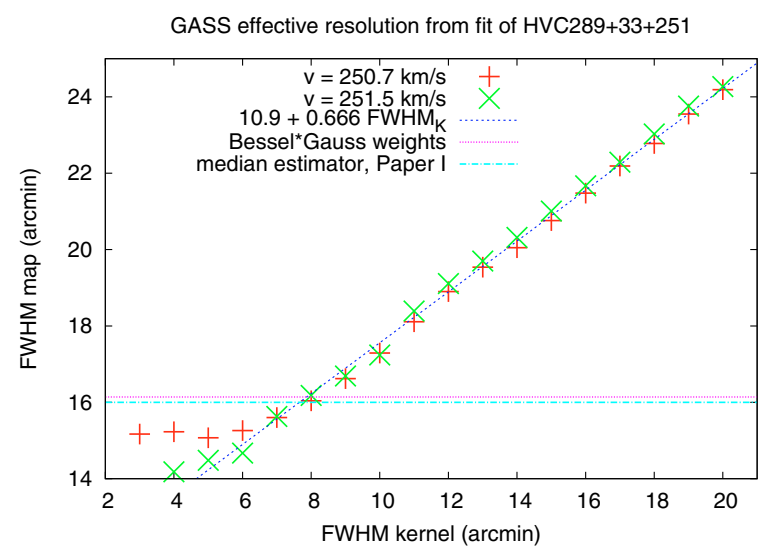

Fig. 6. Effective $F W H M$ resolution $W$ of GASS images as a function of the user-definable Gaussian interpolation kernel $W_{\mathrm{k}}$. For of $W_{\mathrm{k}}>7^{\prime}$ we find $W \sim 10^{\prime} 9+0.666 W_{\mathrm{k}}$. For comparison we show the effective resolution obtained by median gridding and by Bessel interpolation (horizontal lines).

beam width of $W_{\mathrm{t}}$, and a width $W_{\mathrm{k}}$ of the user defined kernel we expect a resulting $F W H M$ width

$W=\sqrt{W_{\mathrm{s}}^{2}+W_{\mathrm{t}}^{2}+W_{\mathrm{k}}^{2}}$.

We tested this relation by generating a series of images of the ultra-compact high-velocity cloud HVC289+33+251 (Putman et al. 2002; Brüns \& Westmeier 2004). We used a grid spacing of 4.8 , corresponding to $1 / 3$ of the $F W H M$ beam size. This object is isolated and has a $F W H M$ diameter of $W_{\mathrm{s}}=4{ }^{\prime} .4$. Since it is unresolved by the Parkes telescope at $1.4 \mathrm{GHz}$ the effective beam $W$ can easily be fitted. This was done for 18 FITS cubes with $3^{\prime}<W_{\mathrm{k}}<20^{\prime}$ at two velocities, $v=250.7 \mathrm{~km} \mathrm{~s}^{-1}$ and $v=251.5 \mathrm{~km} \mathrm{~s}^{-1}$. We determined first an effective telescope beam width $W_{\mathrm{t}}=\sqrt{W_{\mathrm{k}}^{2}-W_{\mathrm{s}}^{2}}=14^{\prime} .2 \pm 1.0$ from the known width of kernel and source. This value agrees well with the expected average $F W H M$ beam width. The individual beams have $14^{\prime}<F W H M<14^{\prime} .5$, with a mean of $W_{\mathrm{av}}=14$. $^{\prime}$.

Next we deconvolve the observed and fitted FWHM width $W_{\text {obs }}$ of the image for the finite width $W_{\mathrm{s}}=4.4$ of the source, $W=\sqrt{W_{\text {obs }}^{2}-W_{\mathrm{s}}^{2}}$. In Fig. 6 we plot $W$ as a function of the convolving kernel width $W_{\mathrm{k}}$. To a good approximation we find for $W_{\mathrm{k}} \gtrsim 6^{\prime}$ the simple relation $W=10^{\prime} 9+0.666 \cdot W_{\mathrm{k}}$. For a kernel $W_{\mathrm{k}}=8^{\prime}$ we obtain $W=16^{\prime}$, identical with the resolution of the images calculated from weighted median estimators as presented in Paper I.

The noise level of the output map depends on the user defined smoothing kernel. We measured the noise in the same FITS cubes that we used to fit beamwidths, at a velocity of $240 \mathrm{~km} \mathrm{~s}^{-1}$ where neither HI emission nor RFI is visible. Figure 7 gives the results. The noise strongly depends on the smoothing kernel; for $W_{\mathrm{k}}=7$. .0 we obtain an rms brightness temperature noise of $57 \mathrm{mK}$, comparable to the results in Paper I. The corresponding effective resolution is $W=15$ ' 6 .

The noise level depends on the strength of the line emission. Within the Galactic plane for $|b| \lesssim 1^{\circ}$ we find a typical increase by a factor of 1.75 . This approximate upper limit is plotted in Fig. 7. For a detailed map of the uncertainties we refer to the bottom map in Fig. 9. In this case $W_{\mathrm{k}}=12$ '.0 was chosen, resulting in a typical noise level of $35 \mathrm{mK}$. It is obvious that the noise in the final maps is affected by RFI mitigation and observational setup. Overlap regions for the raster chosen are clearly visible.

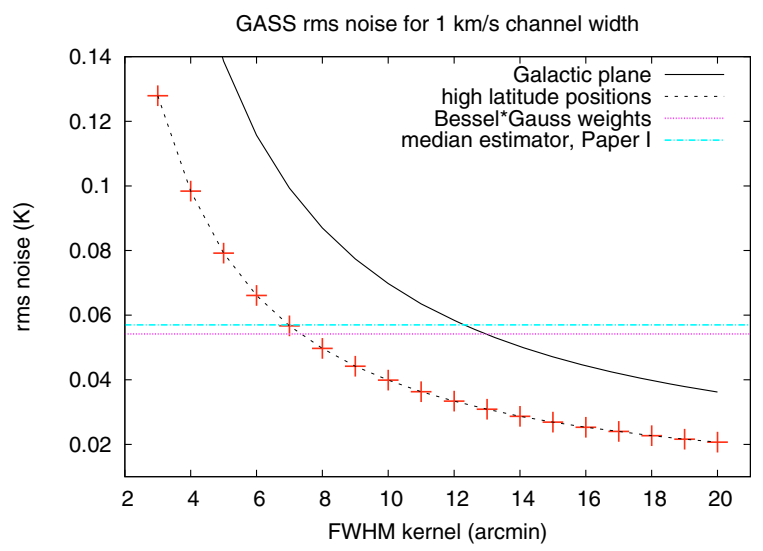

Fig. 7. Effective rms uncertainties of GASS channel maps with $1 \mathrm{~km} \mathrm{~s}^{-1}$ velocity resolution as a function of the user-definable Gaussian interpolation kernel. Crosses indicate fitted values determined at high Galactic latitudes, the solid black line represents upper limits of the noise, valid for most of the positions in the Galactic plane. For comparison we show the effective noise at high latitudes obtained by median gridding and by Bessel interpolation (horizontal lines).

We tested also the optimal gridding of single dish on-thefly observations as proposed by Mangum et al. (2007) with the function

$$
\frac{J_{1}(r / a)}{(r / a)} \exp -(r / b)^{2} \text {. }
$$

$J_{1}$ is the Bessel function, $a=1.55$, and $b=2.52$ for a grid of $1 / 3 F W H M$. This is the default for the AIPS task SDGRD (Greisen 1998). The resulting resolution and noise are plotted as horizontal lines in Figs. 6 and 7. Both median gridding according to Paper I and optimal Bessel filtering are consistent with a Gaussian gridding for a kernel of $W_{\mathrm{k}} \sim 7 ! .5$.

\section{Quality of the data}

To aid in the discussion of this release of the GASS data we have chosen a channel map at $v_{\mathrm{lsr}}=-30.5 \mathrm{~km} \mathrm{~s}^{-1}$ (Fig. 8). At this velocity stray-radiation effects are strong and residual problems easily visible. In the top panel of Fig. 8, we show a map that was generated without a stray-radiation correction but with the instrumental baselines removed as described in Sect. 3. In the middle panel, we plot the map from the final GASS database, corrected for stray-radiation and instrumental baseline. We have chosen a $F W H M$ kernel $W_{\mathrm{k}}=12$ ', resulting in rms uncertainties of $\sim 35 \mathrm{mK}$ at intermediate Galactic latitudes. In the bottom panel of Fig. 8, we show the same channel from the LAB database for comparison. Figure 9 (top) contains the strayradiation contribution removed from the middle map in Fig. 8.

The bottom panel of Fig. 9 displays a compilation of the residual $1 \sigma$ rms uncertainties for a FITS cube generated with $F W H M$ kernel $W_{\mathrm{k}}=12$ ! These uncertainties depend on the integration time and on the system noise. Enhanced noise is caused by low elevation observations, by continuum emission from the Galactic plane, or by RFI mitigation (rejecting data).

The final GASS map, Fig. 8 middle, shows no obvious evidence of residual stray-radiation problems. Time dependence of stray-radiation would cause a correlation with the observational grid that is easily visible in Fig. 9 (bottom). Such residual problems can be seen in the LAB channel map (Fig. 8 bottom). Individual blocks, corresponding to a grid of $5 \times 5$ positions, show up at latitudes of $b \sim-50^{\circ}$, a problem already 
A\&A 521, A17 (2010)

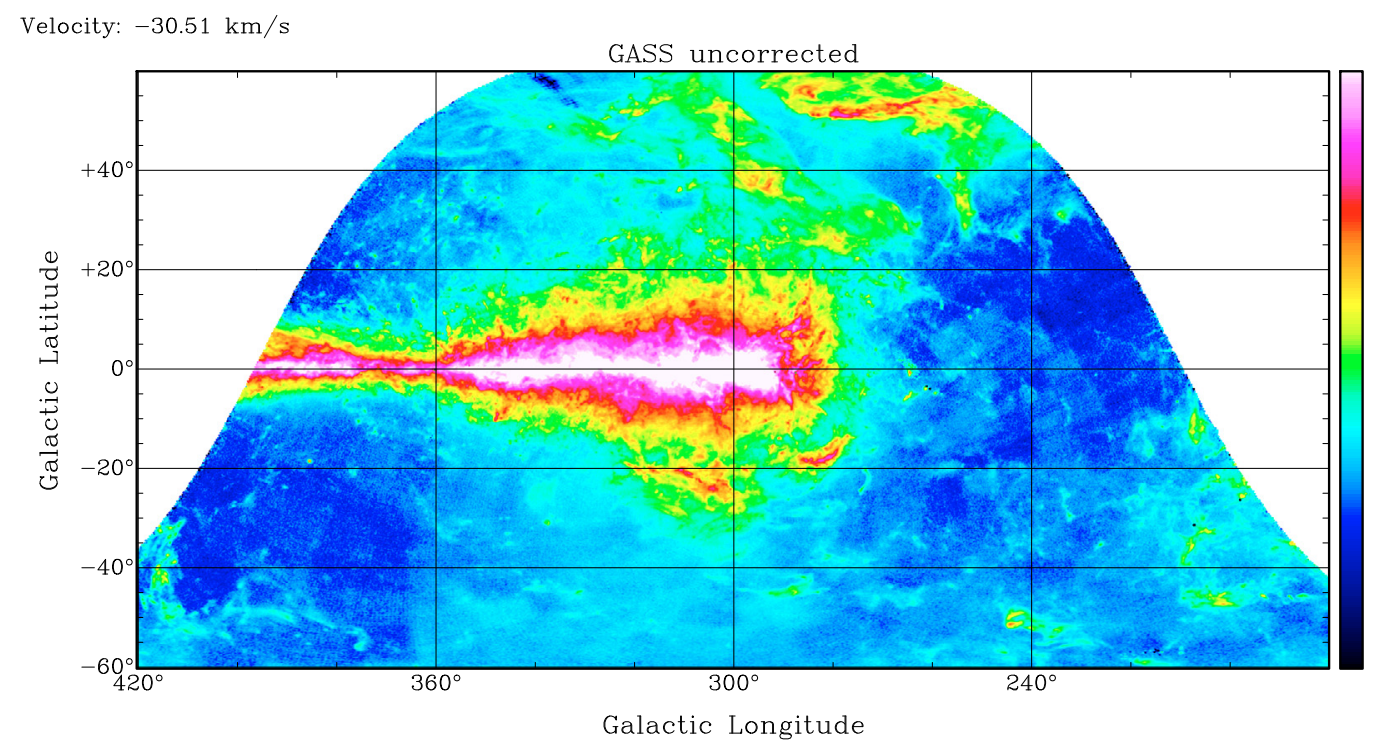

Velocity: $-30.51 \mathrm{~km} / \mathrm{s}$
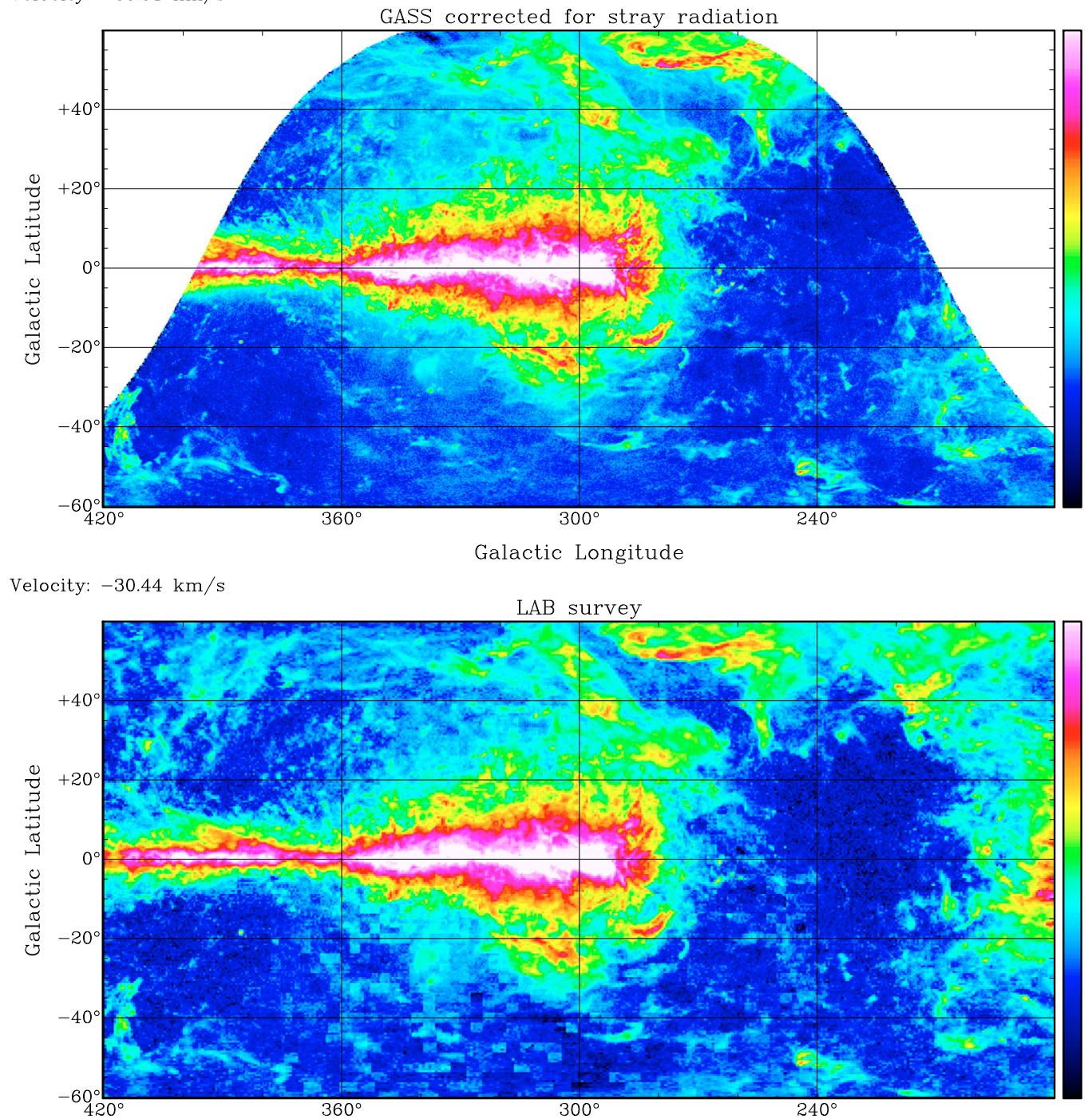

Galactic Longitude

Fig. 8. HI emission at $v_{\mathrm{lsr}}=-30.5 \mathrm{~km} \mathrm{~s}^{-1}$. The intensity scale for the range $-0.12<T<50 \mathrm{~K}$ is logarithmic, emphasizing low level emission. Top: GASS, corrected for instrumental baseline but without stray-radiation correction, middle: GASS corrected for stray-radiation and instrumental baseline, bottom: LAB survey for comparison, showing faint patchy spurious features that do not appear in the new GASS data, e.g. at $l=335^{\circ}$, $b=-40^{\circ}$. 
Velocity: $-30.51 \mathrm{~km} / \mathrm{s}$

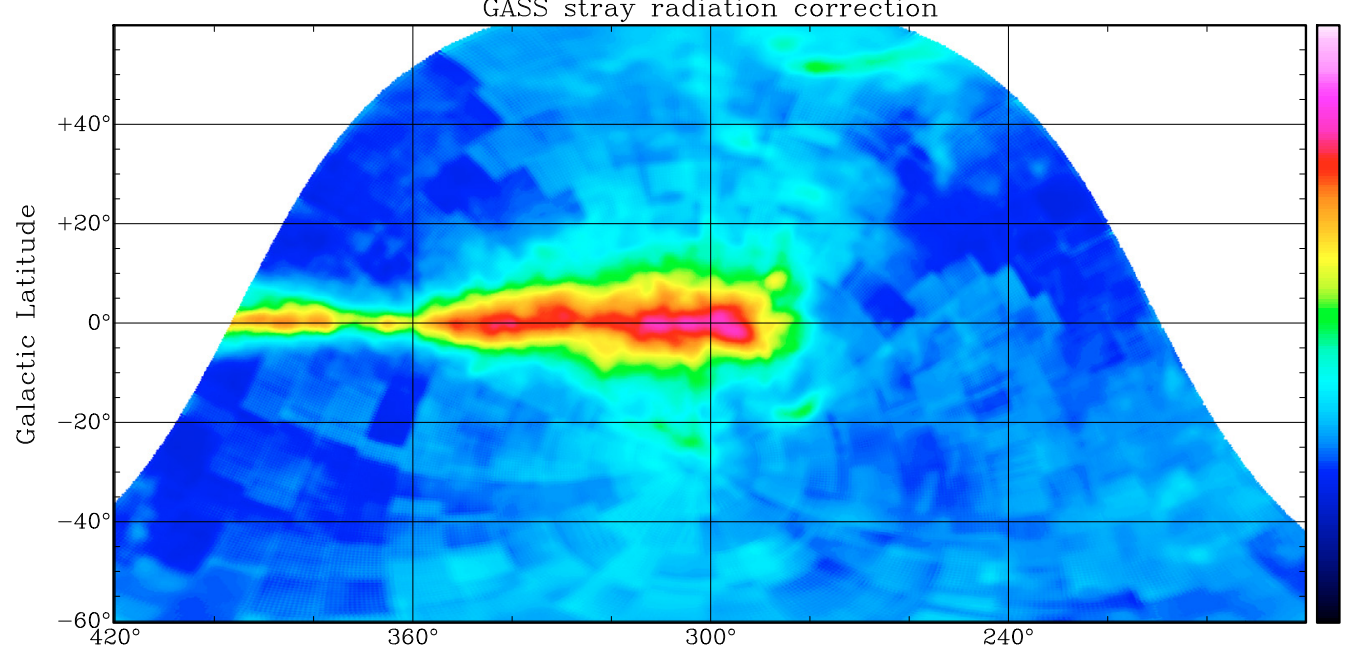

Galactic Longitude

Velocity: $+98.94 \mathrm{~km} / \mathrm{s} \quad$ rms uncertainties for a channelwidth of $1 \mathrm{~km} / \mathrm{s}$

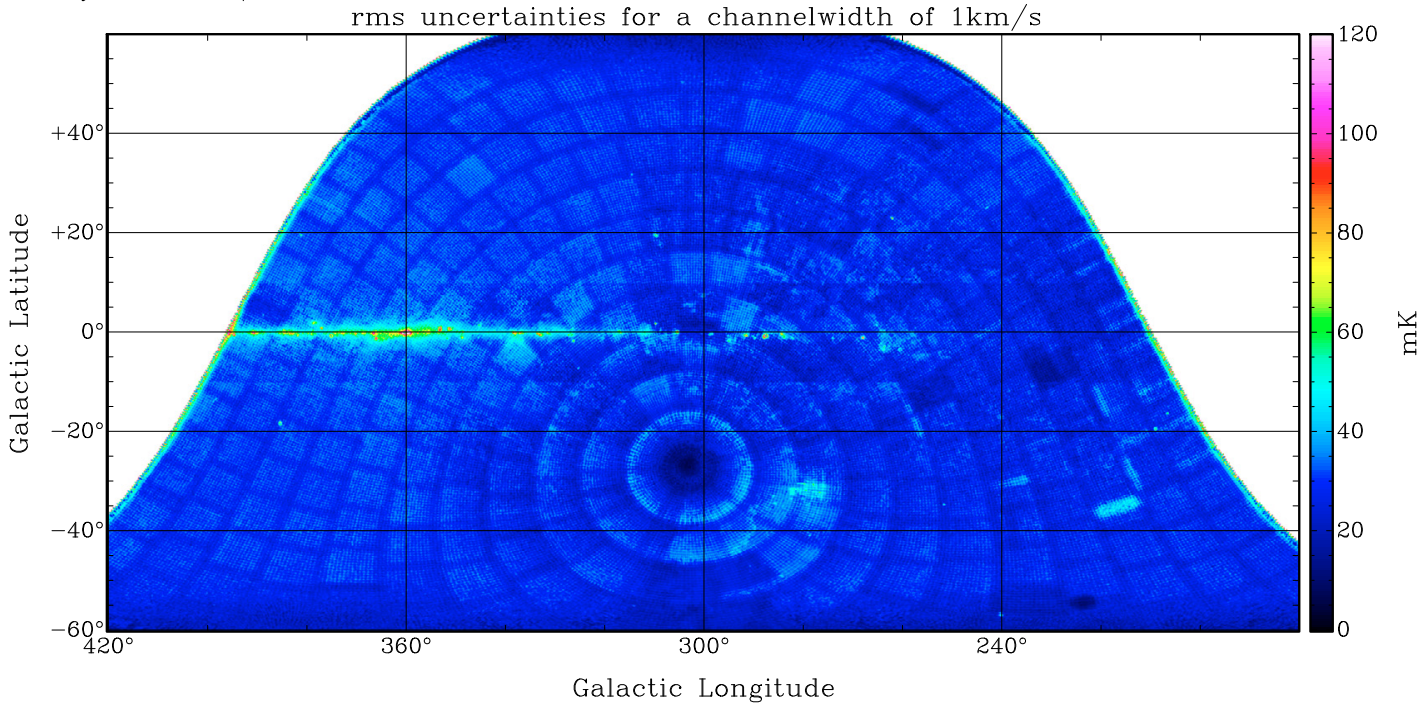

Fig. 9. Top: stray-radiation contribution removed from the GASS at $v_{\mathrm{lsr}}=-30.5 \mathrm{~km} \mathrm{~s}^{-1}$, the intensity scale is identical to Fig. 8 . Bottom: rms noise in the final GASS data, corresponding to the intensity map shown in the central panel of Fig. 8.

mentioned by Kalberla et al. (2005). Similar block structures existed also in the first version of the Leiden/Dwingeloo Survey (Hartmann \& Burton 1997; Hartmann et al. 1996) but could be removed by an improved stray-radiation correction in the second data release of the LAB (Kalberla et al. 2005). Time variability is caused predominantly by a seasonal variation of the strayradiation and needs to be minimized by the correction (Kalberla et al. 1980a,b). The final GASS database is much less affected by residual instrumental problems than the LAB survey. This implies in particular that there was no error propagation from the LAB to the GASS. We conclude that our corrections successfully removed most of the sidelobe influences, remaining systematical errors are probably below $1 \%$. The performance of the survey is equivalent to observations with a telescope having $\gtrsim 99 \%$ main beam efficiency. Scale uncertainties, caused by gain fluctuations, are also probably below 1\% (Sect. 4).

The top panel of Fig. 8, shows that stray-radiation can not be removed with only our baseline correction algorithm as most of the stray-radiation remains in the map. This also demonstrates that our baselining algorithm is safe in the sense that it does not noticeably affect extended profile wings.
We searched for remaining systematical uncertainties in the instrumental baseline. Some residual uncertainties caused by RFI are visible in individual channel maps. In most cases such defects remain on average below $40 \mathrm{mK}$ but there are a few more serious cases. In critical cases we recommend comparing GASS data with the LAB database. Also a comparison of the cleaned database with the dirty data, offered by the web interface, may be helpful.

\section{Data products}

Data from the first release are accessible at http: //www . atnf. csiro.au/research/GASS/Data.html. We provide a web interface at http://www.astro.uni-bonn.de/hisurvey for the second release to retrieve spectra and column densities for individual positions or complete FITS cubes. The user can specify Galactic or Equatorial coordinates, the velocity range, and the FWHM of the smoothing kernel for the optimal resolution. Default values for the gridding are provided as well. Some restrictions to the sizes of the FITS cubes apply, and we currently provide maps only in Cartesian projection. 
The user can check the data for residual contaminations by RFI or stray-radiation by downloading FITS cubes with dirty data (corrected for baseline and stray-radiation but no RFI filtering applied) or stray-radiation corrections that were subtracted as described in Sect. 2. Data at high velocities $\left|v_{\text {lsr }}\right| \gtrsim 160 \mathrm{~km} \mathrm{~s}^{-1}$ can be generated alternatively from both IFs or from single "ghost-free" IFs only (see Sect. 5.6). In the latter case the rms noise for channels at velocities $\left|v_{\mathrm{lsr}}\right| \gtrsim 160 \mathrm{~km} \mathrm{~s}^{-1}$ increases by a factor of $\sqrt{2}$.

Despite all efforts some instrumental problems may still exist in the second data release. Our web based policy for the on-thefly generation of FITS cubes allows appropriate corrections. We ask users of the GASS data to report problems. Information on updates of the database will be provided on the web page.

\section{Summary}

The Parkes Galactic All-Sky Survey (GASS) measured the HI emission in the Southern sky with complete sampling at all declinations $\delta \leq 1^{\circ}$ using the Parkes Radioe Telescope. The survey has an effective angular resolution of 14.'4 at a velocity resolution of $1.0 \mathrm{~km} \mathrm{~s}^{-1}$, and the data were obtained in a way that is sensitive to extended diffuse emission as well as compact sources. The GASS database contains $2.8 \times 10^{7}$ individual spectra with $5 \mathrm{~s}$ integration time observed by on-the-fly mapping. The first data release together with a detailed description of the survey goals and techniques was given in Paper I. Here we focus on the post processing to correct instrumental effects including stray-radiation, radio frequency interference, and residual instrumental spectral baselines.

The GASS is the most sensitive, highest angular resolution survey of Galactic Hi emission ever made of the Southern sky. After correcting for instrumental effects and RFI the GASS data are in excellent agreement with the LAB survey. This is impressively demonstrated with Fig. 8. FITS data cubes are available on request from http: //www . astro. uni-bonn. de/ hisurvey. Our software allows a flexible generation of FITS datacubes with effective resolutions $16 \lesssim F W H M \lesssim 25^{\prime}$ (Fig. 6). The corresponding rms noise fluctuations at high Galactic latitudes are in the range $60 \gtrsim \sigma \gtrsim 20 \mathrm{mK}$ (Fig. 7). We provide clean maps as the final product from our data processing but also maps without RFI elimination. To allow estimates on possible residual stray-radiation effects it is also possible to extract the corrections which were applied.

Acknowledgements. We thank Butler Burton for a very thorough refereeing of this paper with constructive criticism and many valuable suggestions. The GASS would have not been possible without support from the ATNF staff at Parkes and Sydney. We greatly acknowledge assistance in determining the properties of the antenna pattern from M. Kesteven, M. Price, J. Reynolds, and W. Wilson. P. Müller (MPIfR Bonn) provided software for baseline fitting. This project was supported by Deutsche Forschungsgemeinschaft, DFG grant KA1265/5-2. P.K. acknowledges also support during a productive stay in Sydney as a distinguished visitor of the ATNF. The Parkes Radio Telescope is part of the Australia Telescope which is funded by the Commonwealth of Australia for operation as a National Facility managed by CSIRO. D.J.P. acknowledges partial support for this project from NSF grant AST0104439 and from an international faculty development grant from West Virginia University. He thanks the ATNF for its generosity and hospitality during his visit in November 2009 to work on this paper. The National Radio Astronomy Observatory is operated by Associated Universities, Inc., under a cooperative agreement with the National Science Foundation.

\section{References}

Bajaja, E., Arnal, E. M., Larrarte, J. J., et al. 2005, A\&A, 440, 767 Barnes, D. G., Staveley-Smith, L., de Blok, W. J. G., et al. 2001, MNRAS, 322, 486

Brüns, C., \& Westmeier, T. 2004, A\&A, 426, L9

Brüns, C., Kerp, J., Staveley-Smith, L., et al. 2005, A\&A, 432, 45

Burton, W. B. 1985, A\&AS, 62, 365

Dickey, J. M., \& Lockman, F. J. 1990, ARA\&A, 28, 215

Greisen, E.W. 1998, AIPS CookBook, NRAO Socorro, NM

Hartmann, D., \& Burton, W. B. 1997, Atlas of Galactic Neutral Hydrogen (Cambridge: Cambridge University Press)

Hartmann, D., Kalberla, P. M. W., Burton, W. B., \& Mebold, U. 1996, A\&AS, 119,115

Heiles, C., \& Habing, H. J. 1974, A\&AS, 14, 1

Higgs, L. A., \& Tapping, K. F. 2000, AJ, 120, 2471

Kalberla, P. M. W., \& Kerp, J. 2009, ARA\&A, 47, 27

Kalberla, P. M. W., Mebold, U., \& Reich, W. 1980a, A\&A, 82, 275

Kalberla, P. M. W., Mebold, U., \& Velden, L. 1980b, A\&AS, 39, 337

Kalberla, P. M. W., Mebold, U., \& Reif, K. 1982, A\&A, 106, 190

Kalberla, P. M. W., Burton, W. B., Hartmann, D., et al. 2005, A\&A, 440, 775

Kerp, J. 2003, Astron. Nachr., 324, 69

Kerr, F. J., Bowers, P. F., \& Henderson, A. P. 1981, A\&AS, 44, 63

Lockman, F. J. 2002, in Single-Dish Radio Astronomy: Techniques and Applications, ed. S. Stanimirovic, D. Altschuler, P. Goldsmith, \& C. Salter, ASP Conf. Proc., 278, 397

Lockman F. J., \& Condon, J. J. 2005, AJ, 129, 1968

Lockman, F. J., Jahoda, K., \& McCammon, D. 1986, ApJ, 302, 432

Mangum, J. G., Emerson, D. T., \& Greisen, E. W. 2007, A\&A, 474, 679

McClure-Griffiths, N. M., Pisano, D. J., Calabretta, M. R., et al. 2009, ApJS, 181, 398 (Paper I)

Peek, J. E. G., \& Heiles, C. 2008, [arXiv: 0810. 1283]

Peirce, B. 1852, AJ, 2, 161

Prestage, R. M., Constantikes, K. T., Hunter, T. R., et al. 2009, IEEE Proc., 97, 1382

Putman, M. E., de Heij, V., Staveley-Smith, L., et al. 2002, AJ, 123, 873

Robishaw, T., \& Heiles, C. 2009, PASP, 121, 272

Schlegel, D. J., Finkbeiner, D. P., \& Davis, M. 1998, ApJ, 500, 525

Stark, A. A., Gammie, C. F., Wilson, R. W., et al. 1992, ApJS, 79, 77

van Woerden, H. 1962, De neutrale waterstof in Orion, Ph.D. Thesis, Groningen,

Rijksuniversiteit

Weaver, H., \& Williams, D. R. W. 1973, A\&AS, 8, 1

Williams, D. R. W. 1973, A\&AS, 8, 505

Winkel, B., Kalberla, P., Kerp, J., \& Flöer, L. 2010, ApJS, 188, 488 